\title{
Escolas de saúde e inovação tecnológica: desenho de novo dispositivo para acesso venoso
}

\author{
Schools of health and technological innovation: design of new device for venoso access
}

Escuelas de salud e innovación tecnológica: diseño de nuevo dispositivo para el acceso venoso

Sandra Inês Lins de Abreu Mendes ${ }^{1 *}$, José Hamilton Mendes ${ }^{1}$, Samara Abreu Mendes².

\section{RESUMO}

Objetivo: Apresentar desenho para possível confecção de um novo modelo de cateter venoso central e de linha média por inserção periférica. Métodos: Para o embasamento teórico pesquisou-se na internet assuntos referentes a educação, inovação tecnológica, saúde e veias. E na construção do desenho de dispositivo venoso usamos a pesquisa-ação, uma metodologia a qual o investigador se envolve diretamente com o alvo do estudo para que ocorra uma mudança no meio onde ocorre a situação problema. Resultados: Uma contribuição para inovação tecnológica da saúde com a criação de um desenho para possível dispositivo venoso e sua usabilidade com deposito no Instituto Nacional da Propriedade Industrial. Conclusão: Neste trabalho sugerimos melhoria na tecnologia de um dispositivo venoso central e/ou de linha média por acesso periférico para ser usado por enfermeiros e médicos com habilidades e treinamentos em punções venosas periféricas nas infusões venosas de curto a longo prazo durante agravos da saúde do recém-nascido ao idoso, respeitando as condições de rede venosa do paciente em serviços pré-hospitalar, hospitalar e domicílio.

Palavras-chave: Educação, Inovação tecnológica, Saúde, Veias.

\begin{abstract}
Objective: To present a design for possible manufacture of a new model of central venous and midline catheter by peripheral insertion. Methods: For the theoretical basis, subjects related to education, technological innovation, health and veins were searched on the internet. And in the construction of the design of the venous device, we use action research, a methodology in which the researcher is directly involved with the target of the study so that a change occurs in the environment where the problem situation occurs. Results: A contribution to health technological innovation with the creation of a design for a possible venous device and its usability with a deposit at the National Institute of Industrial Property. Conclusion: In this work we suggest an improvement in the technology of a central venous and / or midline device for peripheral access to be used by nurses and doctors with skills and training in peripheral venipuncture in short-term and long-term venous infusions during health conditions of the patient. newborn to the elderly, respecting the conditions of the patient's venous network in pre-hospital, hospital and home services.
\end{abstract}

Keywords: Education, Technological innovation, Health, Veins.

\section{RESUMEN}

Objetivo: Presentar un diseño para la posible fabricación de un nuevo modelo de catéter venoso central y de línea media por inserción periférica. Métodos: Para la base teórica se buscaron en internet temas relacionados con educación, innovación tecnológica, salud y venas. Y en la construcción del diseño del

\footnotetext{
${ }^{1}$ Facultad Interamericana de Ciencias Sociales, Assunção - PY. *E-mail: sandra100abreu@hotmail.com

2 Instituto de pós-graduação e graduação (IPOG), Recife - PE.
} 
dispositivo venoso, utilizamos la investigación acción, metodología en la que el investigador se involucra directamente con el objetivo del estudio para que se produzca un cambio en el entorno donde se produce la situación problemática. Resultados: Un aporte a la innovación tecnológica en salud con la creación de un diseño para un posible dispositivo venoso y su usabilidad con depósito en el Instituto Nacional de Propiedad Industrial. Conclusión: En este trabajo sugerimos una mejora en la tecnología de un dispositivo venoso central y / o de línea media para acceso periférico para ser utilizado por enfermeras y médicos con habilidades y entrenamiento en punción venosa periférica en infusiones venosas de corto y largo plazo durante las condiciones de salud del paciente. recién nacidos a ancianos, respetando las condiciones de la red venosa del paciente en los servicios pre hospitalarios, hospitalarios y domiciliarios.

Palabras clave: Educación, Innovación tecnológica, Salud, Venas.

\section{INTRODUÇÃO}

A educação superior possui uma organização social, disponibilizando oportunidades de crescimento intelectual e científico para toda a população, caracterizada pelo importante papel estável e durável em sua comunidade. As Instituições de Ensino Superior devem estar baseadas em um ideal integrador entre a qualificação dos alunos e o incentivo ao desenvolvimento da nação (CAZAROTTI MLB, 2019).

Assim sendo a Nova Base Nacional Comum Curricular explica que essas competências devem ser trabalhadas com os discentes para que eles possam; analisar situações-problema e avaliar aplicações do conhecimento científico e tecnológico e suas implicações no mundo, propondo soluções que considerem demandas locais, regionais e/ou globais, e comunicar suas descobertas e conclusões a públicos variados (DA SILVA, 2019).

As inovações tecnológicas nas formações acadêmicas dos profissionais de saúde são indicativas para criações e modificações de instrumentos de trabalho visando soluções, facilidade, custo-benefício, tempo resposta, efetividade e segurança na prática da saúde (DO AMARAL PS, 2018).

Para os estudantes da área da saúde, as notícias são bastante positivas, haja vista que o próprio mercado de trabalho sinaliza com a adoção cada vez mais intensa da tecnologia. E começando dentro da sala de aula, a continuidade da adoção das novas formas de acesso à informação continuará se dando de forma natural. A opção da mudança, da adoção do long life learning; fica a critério de cada um (PESSONE A e GOULART E, 2015).

As tecnologias da saúde são descritas em três formas: dura representada pelo material concreto como equipamentos, mobiliário permanente ou de consumo; tecnologia levedura, inclui os saberes estruturados representados pelas áreas a exemplo da clínica médica, odontológica, epidemiológica, entre outras e a tecnologia leve, que se expressa como o processo de produção da comunicação, das relações, de vínculos que conduzem ao encontro do usuário com necessidades de ações de saúde. A temática tecnologia não deve ser tratada através de uma concepção reducionista ou simplista, associada somente as máquinas (MERHY EE e BATISTA T, 2014; DA SILVA BA, et al., 2017).

No Brasil a portaria N.1.122 do Ministério da Ciência, tecnologia, Inovações e Comunicações (MCTIC), de 19 de março de 2020, definem como prioritários projetos de pesquisa, desenvolvimento e inovações voltados às áreas de tecnologias: estratégicas; Habilitadoras; de produção; para Desenvolvimento sustentável; e para qualidade de vida está última envolve os setores da saúde, saneamento básico, segurança hídrica e tecnologias assistivas (MINISTÉRIO DA CIÊNCIA, TECNOLOGIA, INOVAÇÕES E COMUNICAÇÕES BR, 2020).

A inovação na saúde envolve um campo de estudos e capacidade para mobilizar infraestrutura de Ciência, Tecnologia e Inovação (CT\&I/S). Processo político e social, parte do planejamento estratégico do país, visando à redução nacional da dependência de insumos para saúde produzidos no exterior e reforça a economia política do país (TENÓRIO MM, et al., 2017). 
A lei 13.243/2016 e decreto 9.283/2018, marcos legais da (CT\&I/S) desburocratiza a pesquisa e inovação no Brasil, incentiva e interage com as instituições cientificas, tecnológicas, as universidades, serviços de produção e estado (GOMES RADOS e TEIXEIRA CS, 2018).

Tecnologias em saúde são necessárias para recuperação e manutenção da vida em pacientes críticos, já que estes dispositivos acabam sendo extensão do próprio corpo dos pacientes, em contrapartida podem causar infecção e colocar em risco essa clientela caso não sejam utilizadas de maneira adequada (SILVA EA e DA CRUZ IC, 2018).

No caso dos cateteres venosos, os mais usados são: cateter periférico curto (jelco) de curta permanência; cateter para hemodiálise pode ser periférico se for de curta permanência ou central se for de longa permanência; cateter vascular central; peripherally inserted central venous catheter ou o cateter vascular central de inserção periférica; cateter semi implantado; cateter totalmente implantado para longa permanência e o cateter umbilical instalados em recém nascidos nas primeiras horas de vida (OLIVEIRA CG e RODAS ACD, 2017).

Existem complicações que envolvem cateteres periféricos curtos e afeta significativamente os custos dos cuidados de saúde, a qualidade de vida dos pacientes, morbidade, mortalidade e aumento das despesas com o tratamento, principalmente quando a internação é prolongada, este é de curta permanência não atendendo as necessidades de todas as infusões (ANSEL B, et al., 2017).

Os cateteres venosos centrais por punção de tórax nas veias jugulares interna direita e esquerda e subclávia desempenham um papel importante no tratamento de pacientes hospitalizados, especialmente aqueles em estado crítico. Utiliza-se como procedimentos diagnósticos e dispositivos invasivos que podem desencadear complicações durante o procedimento e as Infecções Relacionadas à Assistência à Saúde (IRAS) (PERIN DC, et al., 2016).

Cateter Central de Inserção Periférica (CCIP) dispositivos de acesso venoso central, caso não ficando sua extremidade em veias centrais ficará em linha média tornando também eficientes para administrações de medicações, hemoderivados e coletas de sangue. Seu uso diminui o custo do procedimento para o serviço, quando comparado com outros tipos de cateteres centrais que são realizados sob o controle de outros profissionais como anestesistas, cirurgiões, radiologistas. Outras vantagens do CCIP são as referidas pelos pacientes, sendo uma das mais importantes o relato de dor mínima na hora da inserção ou remoção (SANTOLIM TQ, 2017).

São fatores desencadeadores de reações adversas: tipo de material do cateter, calibre, comprimento e espessura, número de inserções em um mesmo paciente, local de inserção e período de manutenção do cateter (MENSOR LP, et al., 2016). Os Cateteres Centrais de Inserção Periféricas (CCIPs) em material de silicone são hemocompatíveis e menos trombogênicos, dificultando à adesão de microrganismos em suas paredes, o que permite uma permanência prolongada variando de semanas até seis meses, pouco acessíveis nos serviços de saúde do Brasil (SWERTS CAS, et al., 2020).

Quando se trata de acessibilidade, considera-se também à disponibilidade de recursos em dado tempo e lugar; nesse caso, temos os aspectos dos serviços que exercem, impactam e repercutem na possibilidade de uso pela população (CARVALHO MEL, et al., 2020). A incorporação de novas tecnologias na área da saúde presente nos dias atuais, embasada nos avanços do conhecimento científico e nas demandas da sociedade, em busca do aprimoramento diagnóstico e de uma melhoria na prestação de assistência à saúde (OLIVEIRA CG e RODAS ACD, 2017).

Verifica-se a importância de o Estado implantar políticas públicas que reconheçam a relação entre saúde, tecnologia industrial e desenvolvimento com o apoio da sociedade civil, a fim de que haja base social e sustentação política objetivando responder aos problemas epidemiológicos do país e viabilizar a combinação entre inovação e bem-estar social (FERNANDES DRA, 2020).

Existe uma tecnologia evolucionária, inserindo melhorias nos produtos/serviços embora a repercussão da lacuna gerada pela sua aplicabilidade faça com que o (a) uso/manutenção dessas tecnologias possa gerar 
dificuldades em seu manuseio quando não vinculado à capacitação e educação permanente (KREMPSER P, et al., 2017).

O objetivo desse trabalho foi: apresentar desenho para possível confecção de um novo modelo de cateter venoso central e de linha média por inserção periférica.

\section{MÉTODOS}

Este trabalho foi desenvolvido por três autores sendo dois enfermeiros e um arquiteto. Para o embasamento teórico pesquisamos e estudamos em artigos, livros e dissertações disponíveis na internet nas plataformas do Google escolar e Web artigos usando as seguintes palavras chaves: Educação, Inovação tecnológica, Saúde e Veias. Os critérios de escolha destas referências foram as dos últimos seis anos, isto é, de 2014 a 2020 no total de 24 bibliografias pesquisadas e referenciadas.

O desenho foi realizado em programas de computador pelo profissional arquiteto também participante deste estudo, seguindo as descrições orientada pelos enfermeiros do estudo para a construção do desenho de possível novo modelo de dispositivo venoso e sua usabilidade e apresentados em figuras e tabela que consta nos resultados deste trabalho.

A ideia do desenho desse possível novo modelo de Cateter Venoso Central e de Linha Média por Inserção Periférica apresentado neste estudo, partiu dos problemas identificados com os cateteres venosos existentes no mercado da saúde durante experiência na prática da enfermagem em emergências e cuidados intensivos identificados pelos enfermeiros autores.

Embasado pela metodologia e orientação da pesquisa-ação que é um tipo de pesquisa de campo em que o investigador se envolve diretamente com o objeto do estudo, havendo uma interferência do pesquisador para que ocorra uma mudança no meio onde ocorre a situação problema.

\section{RESULTADOS E DISCUSSÃO}

Os resultados do material escrito na parte teórica deste trabalho deram respaldo sobre a importância da inovação tecnológica no ensino dos cursos da saúde para contribuição e incentivos a melhoria e criação de suprimentos da saúde e corrobora com as identificações das dificuldades encontradas com os dispositivos venosos mais utilizados atualmente.

Foi utilizado o método da pesquisa ação que defende a resolução de problemas, neste caso os dispositivos venosos mais utilizados pelos enfermeiros na assistência à saúde. Construímos o desenho de um possível novo modelo de Cateter Central e de Linha Média de Inserção Periférica apresentados nas Figuras 1 e 2 e a usabilidade deste, demostrada na Tabela 1.

Cateteres venosos central e de linha média refere-se ao posicionamento de um dispositivo apropriado para acesso vascular cuja extremidade atinja os grandes vasos centrais como aorta e veias cavas superiores e inferiores, independentemente do local da inserção periférica, ficando sua extremidade central ideal para administração de qualquer tipo de solução endovenosa e também para medidas de pressão venosa central (PVC). Esse mesmo cateter introduzido em uma veia periférica e não alcançando as veias centrais é nomeado de linha média indicado nas emergências para administração de grandes volumes e medicações indispensáveis no momento do evento (MELO JSA e MENDES SILA, 2019).

O fortalecimento da ideia do desenho para uma possível confecção industrial de novo modelo de cateter central e de linha média por inserção periférica poderá ter ganhos em custos e benefícios. Deverá ser aplicado por enfermeiros e médicos habilitados e treinados para essa modalidade facilitando o atendimento nas necessidades de administrar qualquer tipo de infusões a pacientes com agravo a saúde de curto a longo prazo em tratamento até mesmo nas urgências e emergências das diversas unidades de assistência à saúde com propósito de diminuir danos ao cliente, profissionais e empresas de saúde. 
Figura 1 - Vista frontal do conjunto que forma o mandril ou introdutor composto por: agulha com bainha bipartida, regulador, tampa protetora, área de refluxo e regulador.

\section{VISTA \\ FRONTAL}

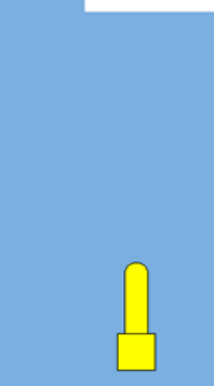

TAMPA PROTETORA

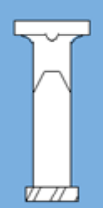

ÁREA DE REFLUXO

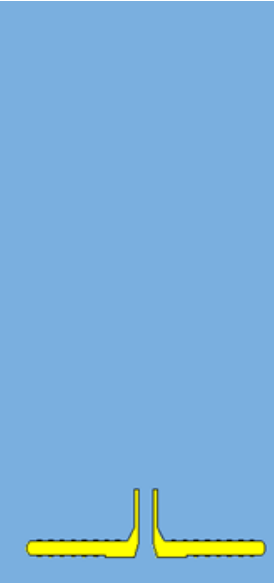

REGULADOR

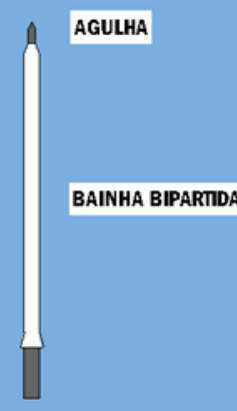

AGULHA

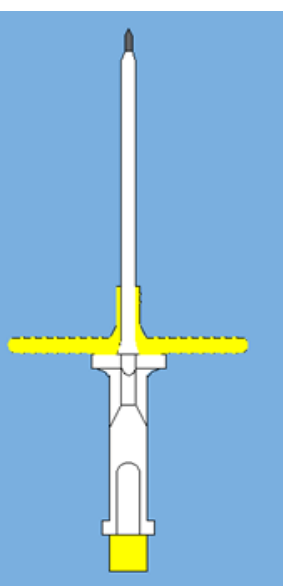

AGULHA E MANDRIL

Fonte: Mendes SILA, et al., 2020.

O introdutor parte do cateter responsável pela punção da veia, que é a introdução de uma agulha metálica desde a pele até o interior do vaso venoso dando abertura para em seguida ocorrer a introdução do cano ou cateter. A agulha deverá ser revestida por bainha bipartida que após a introdução do cateter até a sua medida final está bainha é partida e retirada.

Figura 2 - Variações de tamanho, diâmetro e cores dos possíveis modelo de cateter venoso central e de linha média por inserção periférica representados.

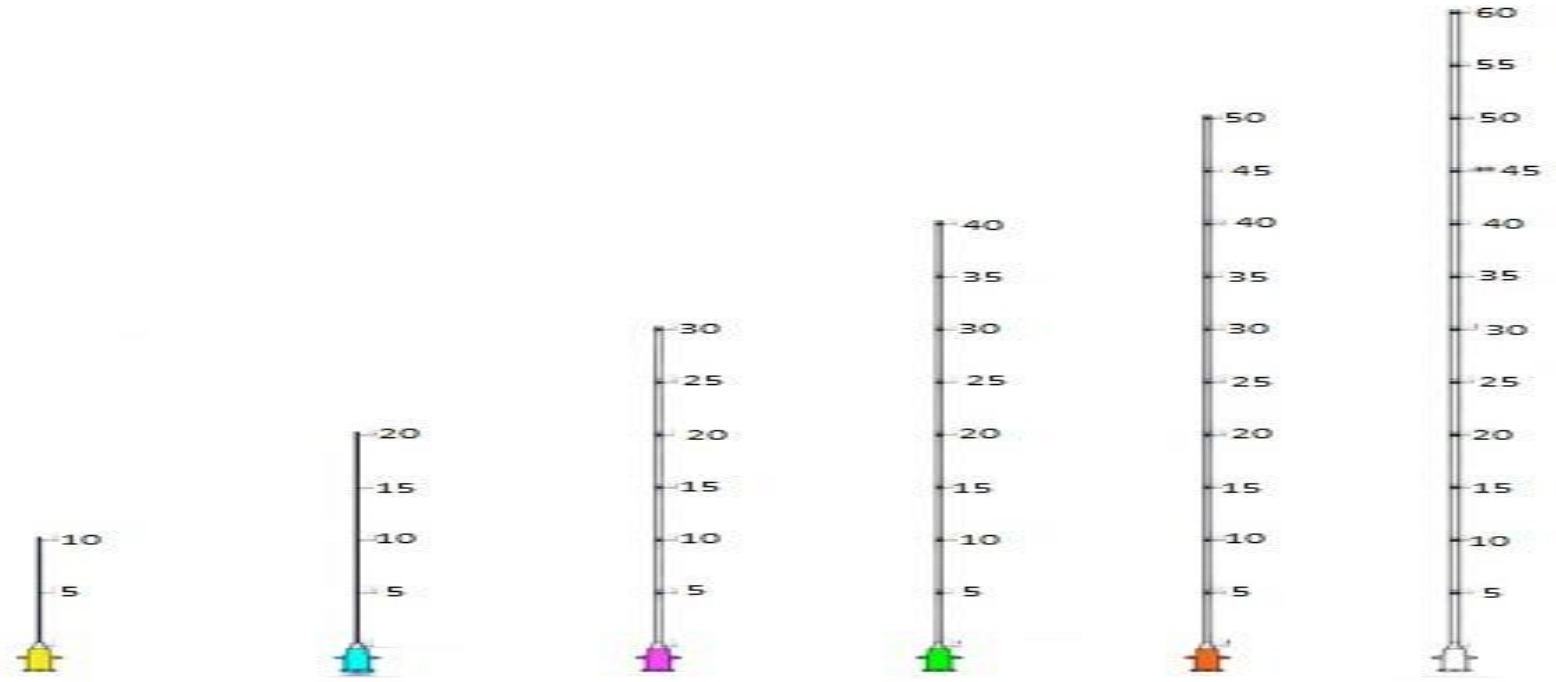

Fonte: Mendes SILA, et al., 2020.

A padronização desse desenho de cateter deverá ser em cores, tamanhos e diâmetro definidos pelos autores deste trabalho idealizando o modelo de possível novo Cateter Central e de Linha Média por Inserção Periférica. Deverão estar dispostos em 6 tamanhos de 10 a 60 centímetros, nas cores (amarela, azul, rosa, verde, laranja e branco) e espaço interno de 1 a 6 french ou diâmetros de $(0,333 \mathrm{~mm} ; 0,6667 \mathrm{~mm} ; 1 \mathrm{~mm}$; $1,36 \mathrm{~mm} ; 1,67 \mathrm{~mm}$ e $2 \mathrm{~mm}$ ). Nesta imagem destacam-se as cores em neon que são um diferencial significativo neste produto com o objetivo de facilitar identificação por tamanho e diâmetro do cateter, agilizando a visualização no momento dos procedimentos de acesso venoso. 
Tabela 1 - A usabilidade do possível novo modelo de cateter central e de linha média por inserção periférica representado e demostrado.

\begin{tabular}{|c|c|c|c|c|}
\hline Usabilidade & $\begin{array}{c}\text { Variação } \\
\text { cromática neon }\end{array}$ & $\begin{array}{c}\text { Diâmetro do lúmem } \\
\text { em french }\end{array}$ & $\begin{array}{c}\text { Diâmetro do } \\
\text { lúmem em mm }\end{array}$ & $\begin{array}{c}\text { Comprimento do } \\
\text { cateter }\end{array}$ \\
\hline Neonatal & AMARELO & $1 \mathrm{FRENCH}$ & $0,333 \mathrm{MM}$ & $10 \mathrm{CM}$ \\
\hline Neonatal & AZUL & $2 \mathrm{FRENCH}$ & $0,6667 \mathrm{MM}$ & $20 \mathrm{CM}$ \\
\hline Pediatria & ROSA & $3 \mathrm{FRENCH}$ & $1 \mathrm{MM}$ & $30 \mathrm{CM}$ \\
\hline Pediatria & VERDE & $4 \mathrm{FRENCH}$ & $1,35 \mathrm{MM}$ & $40 \mathrm{CM}$ \\
\hline Adulto & LARANJA & $5 \mathrm{FRENCH}$ & $1,67 \mathrm{MM}$ & $50 \mathrm{CM}$ \\
\hline Adulto & BRANCO & $6 \mathrm{FRENCH}$ & $2 \mathrm{MM}$ & $60 \mathrm{CM}$ \\
\hline
\end{tabular}

Fonte: Mendes SILA, et al., 2020.

Esta referência de usabilidade apresentada na tabela acima em seis tamanhos e diâmetros diferentes é também uma identificação, facilitando o entendimento do possível cateter no momento de escolha em necessidades de acessos venosos nas diferentes idades e onde cada tipo de cateter representado por cores e tamanho variável é escolhido para o uso e prática de acordo com o perfil físico de cada paciente e condições de rede venosa no momento do atendimento de saúde e a depender das condições da emergência e gravidade do paciente.

Compreende-se que na saúde exige qualificação profissional e equipamentos que sejam o mais próximo possível do compatível com a estrutura humana em qualquer idade e situação de saúde e doença. E a inovação tecnológica deve entender sobre o corpo humano tentando e adequando ofertar o produto com melhor qualidade para a saúde humana. É de grande importância à capacitação da equipe por intermédio de educação continuada para o desenvolvimento de competências, habilidades e incentivar a composição do Time de Terapia Intravenosa (TTIV). São ações indispensáveis que favorecem a gestão dos dispositivos intravenosos resultados promissores na qualidade, segurança do paciente e da equipe multiprofissional (OLIVEIRA ES de et al., 2017).

Saúde é um campo vasto para a necessidade de criar, modificar e transformar os subsídios que dispomos e necessitamos para $o$ atendimento dos pacientes. Educadores da saúde possuem chances de observar no dia a dia durante a prática assistencial o que está efetivamente resolvendo e solucionando o problema do paciente sem danos e os que causam falhas impactantes no controle da saúde e doença diante da promoção, prevenção, diagnóstico, tratamento, cura e reabilitação. A comunidade acadêmica da saúde quando observar que determinado protocolo e equipamento empregado no tratamento da saúde está ultrapassado, causando dúvidas, retardo na assistência e falhas diante da necessidade dos clientes é hora de pensar em mudanças e melhoramento para uma assistência mais efetiva com menos riscos e gastos econômicos.

A escola enquanto aquela que instrumentaliza o sujeito por meio da apropriação do conhecimento, provocando uma reflexão crítica da realidade, torna-se espaço de aprendizagens, de vivências significativas, possibilitando o desenvolvimento das capacidades e potencialidades humanas à medida que em sua prática viabiliza o pensar, a participação e a necessidade de mudança (BATISTA EL, 2019).

Complicações de punção venosa é o resultado não desejado de uma terapia recomendada, associada a fatores de riscos como o tempo de terapia, fármacos, características do paciente, habilidade técnica do profissional, dispositivos entre outros, causando efeitos adversos aos pacientes como: inflamações e infecções de corrente sanguínea. Os dispositivos intravasculares são indispensáveis nos cuidados aos pacientes com necessidades de infusões venosas. Os avanços gerados nos sistemas de inovação produzem não só impactos econômicos na sociedade, como impactam na qualidade de vida da população e consequentemente, sobre a capacidade produtiva do país (FERNANDES DRA, 2020).

Cateteres venosos curtos Jelco de uso diário por profissionais da enfermagem durante a assistência nos serviços de saúde pré-hospitalar, hospitalar e ambulatórios com movimentos do paciente e/ou equipe de atendimento é facilmente tracionado com perda do acesso por expulsão, dobras e obstrução impedindo o 
tratamento desejado, não é indicado a receber todos os tipos de infusões, seu tempo de utilidade é no máximo de 96 horas sendo necessário várias punções para trocas do dispositivo caso o paciente necessite de tratamento a longo prazo.

O jelco poderá causar inflamação, flebite e até necrose de tecidos como pele, músculos e tendões; traz ineficiência do tratamento por não assegurar que este cateter ou agulha esteja realmente no interior da veia; dependendo do modo da punção há perfurações na continuidade do vaso acarretando infiltrações para os tecidos; mesmo estes dispositivos ficando no lúmen da veia ocorrem queimaduras e necroses quando medicações administradas possuem substâncias com efeitos vesicantes, erosivas e necrotizante.

Cateteres venosos centrais aplicado por médicos com punção de tórax em veias subclávia e jugular interna durante a punção poderá atingir artérias e pulmões e não são propícios em situações de extremas emergências, o tempo de implantação do mesmo é inconveniente durante colapso cardíaco que não deverá haver interrupção nas massagens cardíacas externas do tórax, sendo necessário acesso venoso seguro para administrações das medicações e eletrólitos.

O cateter venoso central de inserção periférica (CCIP) que progride até o terço distal da veia cava superior ou proximal da veia cava inferior previne complicações iatrogênicas (hemorragias, pneumotórax e hemotórax). Pouco riscos de incidentes durante sua inserção, dispensa ambiente cirúrgico e processo anestésico, podendo ser inserido e removido à beira do leito do paciente pelo profissional Enfermeiro (FALLOUH N, et al., 2015; DI SANTO MK, et al., 2017).

O cateter central e de linha média por inserção periférica deverá estar presente em todos os serviços de saúde de média e alta complexidade, acessível aos profissionais Enfermeiro e Médico habilitados em punções de veias periféricas como jugular externa, veias de membros superiores e membros inferiores em qualquer idade e condições de rede venosa periférica, aumentando as opções de dispositivo de acesso venoso.

O silicone é um polímero que engloba o elemento químico elastômero de silicone unido ao hidrogênio, oxigênio e ao carbono. Presente nas variedades de materiais biomédicos, inclusive na fabricação de cateteres de longa permanência. Apresenta elevada inércia química, biocompatibilidade e força tênsil, resistência à temperatura e oxidação, alta resistência com maior flexibilidade, baixa aderência bacteriana e trombogenicidade (MARINO PL, 2015).

Possíveis confecções do Cateter Central e de Linha Média por Inserção Periférica deverá ser em material de silicone com resistência para suportar altas pressões durante administração de fluídos por diferentes calibres de seringas e volume de soluções por bombas de infusões, sem fio guia, radiopaco, flexível. Facilitando a introdução no leito venoso com ajuda de pinça, sem contato a luva do profissional aplicador.

Procede-se a introdução do cateter propriamente dito com auxílio de pinça delicada com a ponta lisa (para não danificar o cateter) através da bainha introdutora (porta de entrada). Destaca-se que o cateter não deve ser manuseado com as luvas, uma vez que o talco nelas existente pode ser preditivo para complicações químicas. Radiopacidade consiste na capacidade do cateter permitir visualização por intermédio de imagem de raios-X ou fluoroscopia, sendo possível pelo elemento bário (radiopaco) adicionado ao material durante sua fabricação. Essa concentração de bário não pode ser superior a 30\%, do contrário ocasionaria a quebra do material pelo aumento de fragilidade (OLIVEIRA ES de, et al., 2017).

\section{CONCLUSÃO}

Pesquisas com inovações tecnológicas deverá estar presente na comunidade acadêmica da saúde, cooperando no desenvolvimento das melhorias dos suprimentos de saúde existente no mercado e criação de outros equipamentos na intensão de otimizar tempo/resposta, capacidade de ação e custo/ benefício de cada insumos e instrumentos usados para assistência à saúde com requisitos que fará diferença aos profissionais para realização de procedimentos seguros. Sugerimos e apresentamos neste estudo um desenho para possível novo modelo de cateter venoso central e de linha média por inserção periférica que poderá ter aplicação pelos profissionais enfermeiros e médicos com habilidades e treinamentos em punções venosas 
periféricas nas infusões venosas de curto a longo prazo do recém-nascido ao idoso, respeitando as condições de rede venosa nos serviços pré-hospitalar, hospitalar e domicilio com procedimento rápido, sem necessidades de anestesias e menos riscos possíveis.

\section{REFERÊNCIAS}

1. ANSEL B, et al. Aumentando o tempo de permanência do cateter periférico curto. Journal of Infusion Nursing, 2017; 40(3); 143-146.

2. BATISTA EL. Trabalho, Educação e Emancipação Humana. Paco e Littera, 2019.

3. BRASIL. Ministério da Ciência, Tecnologia, Inovações e Comunicações MCTIC no 1.122, portarias 19 de mar. D.O.U. 2020.

4. CARVALHO MEL, et al. A acessibilidade nos serviços de saúde sob a perspectiva da pessoa com deficiência. Revista Eletrônica Acervo Saúde, 2020; 12(1); 1767.

5. CAZAROTTI MLB. As relações entre formação humana e a globalização: Uma análise de educação e trabalho. Revista Eletrônica Acervo Científico, 2019; 2; 325.

6. DA SILVA BA, et al. Dimensões de risco no trabalho da enfermagem: estudo reflexivo. Revista Fluminense de Extensão Universitária, 2017; 7(2).

7. DA SILVA EA. O Ensino de Física e as Energias Renováveis. Revista Acervo Educacional (online); 2019; $1: 1309$.

8. DI SANTO MK, et al. Cateteres venosos centrais de inserção periférica: alternativa ou primeira escolha em acesso vascular? Jornal vascular brasileiro, 2017; 16(2): 104-112.

9. DO AMARAL PS. Complexo Econômico Industrial da Saúde: os reflexos jurídicos dos estímulos ao desenvolvimento tecnológicos previstos na Lei oㅡ 13.243/2016. Cadernos Ibero-Americanos de Direito Sanitário, 2018; 7(1), p. 96-110

10. FALLOUH N, et al. peripherally inserted central catheter-associated deep vein thrombosis: a narrative review. The American journal of medicine, 2015; 128 (7); 722-738.

11. FERNANDES DRA, et al. Contribuições do modelo Fatores Críticos de Sucesso para análise da gestão de Parcerias para o Desenvolvimento Produtivo de um laboratório oficial. Cadernos de Saúde Pública, 2020; 36; 00059219.

12. GOMES RADOS, TEIXEIRA C S. As tipologias de habitats de inovação: uma análise da legislação vigente do sul do Brasil sob luz do novo marco legal de ciência, tecnologia e inovação. REAVI-Revista Eletrônica do Alto Vale do Itajaí, 2018; 7(11); 10-19.

13. KREMPSER P, et al. Evolução do Processo de Punção Venosa Periférica e dos Recursos Tecnológicos Segundo Profissionais de Enfermagem. Cienc Cuid Saude, 2017;16(3).

14. MARINO PL. Compêndio de UTI, $4^{\mathrm{a}}$ ed. Artmed. 2015. Tradução Ane Rose Bolner, Jussara N.T. Burnier, Paulo Henrique Machado. Consultoria, supervisão e revisão técnica desta edição: Rafael Barberena Moraes, Márcio Manosso Boniatti. 108 Obra originalmente publicada sob título "The ICU Book, 4th Edition".

15. MENSOR LL, et al. Custo-efetividade de cateteres periféricos com plataforma de estabilização integrada sob a perspectiva hospitalar no Brasil. JBES: Brazilian Journal of Health Economics/Jornal Brasileiro de Economia da Saúde, 2016; 8(1).

16. MERHY EE, BATISTA T. 'Tecnologia leve' do trabalho vivo em ato', na MERHY, E.E Saúde: a cartografia do trabalho vivo. 2014.

17. MELO JSA DE, MENDES SILA DE. Cateter venoso central e de linha média por inserção periférica. Web artigos, 2019.

18. OLIVEIRA CG, RODAS ACD. Tecnovigilância no Brasil: panorama das notificações de eventos adversos e queixas técnicas de cateteres vasculares. Ciência \& Saúde Coletiva, 2017; 22; 3247-3257.

19. OLIVEIRA ES, et al. Avaliação estatística e protocolo para uso de cateter central de inserção periférica na oncohematologia. Dissertação de Mestrado. Universidade Tecnológica Federal do Paraná; 2017; 161.

20. PERIN DC, et al. Evidências de cuidado para prevenção de infecção de corrente sanguínea relacionada a cateter venoso central: revisão sistemática. Revista Latino-Americana de Enfermagem, 2016; 24.

21. PESSONI A, GOULART E. Tecnologias e o ensino na área da saúde. ABCS Health Sciences, 2015; 40(3).

22. SANTOLIM TQ. Benefícios e riscos do cateter central de inserção periférica (CCIP): experiência em 1023 procedimentos. Tese de Doutorado. Universidade de São Paulo; 2017; 87p.

23. SILVA EA, DA CRUZ ICF. Guidelines for evidence-based practice for the control of nursing intervention in the prevention of central venous catheter-related infection, a challenge in practical ICU care-Systematized Literature Review. Journal of Specialized Nursing Care, 2018; 10(1).

24. SWERTS CAS, et al. A utilização do cateter central de inserção periférica em uma unidade de terapia intensiva neonatal. Revista Eletrônica Acervo Saúde, 2020; 40; 226. 